\title{
INFLUENCE OF ELECTRODE POSITION ON THE ELECTROSPINNING PROCESS
}

\author{
Ondrej BATKA, Josef SKRIVANEK \\ TUL - Technical University of Liberec, Department of Textile Machine Design, Liberec, Czech Republic, EU, \\ ondrej.batka@tul.cz
}

https://doi.org/10.37904/nanocon.2019.8664

\begin{abstract}
The work is focused on the assessment of mutual influence of group of spinning electrodes placed in one plane in dependence on their mutual distance, during AC-electrospinning. From the results of the experiment it was found that the distance between the electrodes affects the stability of the nanofiber production process and the siding behavior while AC-electrospinning. In the first phase of the research, simulations of electric field distribution around spinning electrodes were performed. The results obtained by the research will serve for a suitable choice of design parameters of the device for production of flat nanofibrous textile by the ACelectrospinning method to achieve the required width of the nanofibrous textile.
\end{abstract}

Keywords: Spinning electrode, electrospinning, electric field, nanofiber

\section{INTRODUCTION}

Electrospinning is widely used for the production of nanofibres. This technology offers a wide range of industrial and medical applications in many areas, eg in fluid filtration, electrically conductive nanofibers, battery membranes, nanofiber composites, medicine and wound dressings, tissue engineering materials, materials for drug delivery system [1]. For the implementation of the electrostatic spinning process, the basic accessories are power supply, a spinning electrode, dosing device, a polymer solution and a collector on which formed fibers are trapped [2].

The principle of the electrospinning is based on a high voltage that is applied to the electro-spinnable polymeric solution. The process begins with reaching of a specific value of the electric field strength, which is called critical field intensity value, after overcoming of liquid surface tension. For this purpose many technologies have been developed [3]. AC-electrospinning technology is based on the usage of AC voltage, the voltage is applied to the electrode only [4].

Many applications require a relatively wide layer of the resulting nanofiber product containing nanofibers. This is related to the productivity of the AC spinning process. It is very problematic to achieve a wide layer of nanofibers with only one electrode. In order to ensure the required production, the electrodes can be arranged in different groups to achieve the appropriate productivity and nanofiber product width [3]. It was verified that the electrodes influence at a certain distance in terms of changes in the shape and width of the plume and also in productivity related to the intensity of the electric field [6]. For efficient production of nanofibres in a wide range, it is beneficial to know the distance at which mutual interference among electrodes occurs. Accordingly, by arrangement the electrodes, one wide continuous layer, which could not be produced by only one electrode, can achieve the wide layer of nanofiberes with a minimum number of electrodes.

\section{SIMULATIONS}

The finite element method was used to simulate the distribution of the electric field intensity around the electrodes for several different electrode distances. The electrodes were arranged in one plane with a variable parameter, which was their mutual distance. Figure 1 and Figure 2 show the results of the simulation in which 
among the electrodes there was the lowest and the highest possible mutual technological distance between 160 and $300 \mathrm{~mm}$ [7]. Autodesk Simulation Mechanical 2015 software was used for simulations.

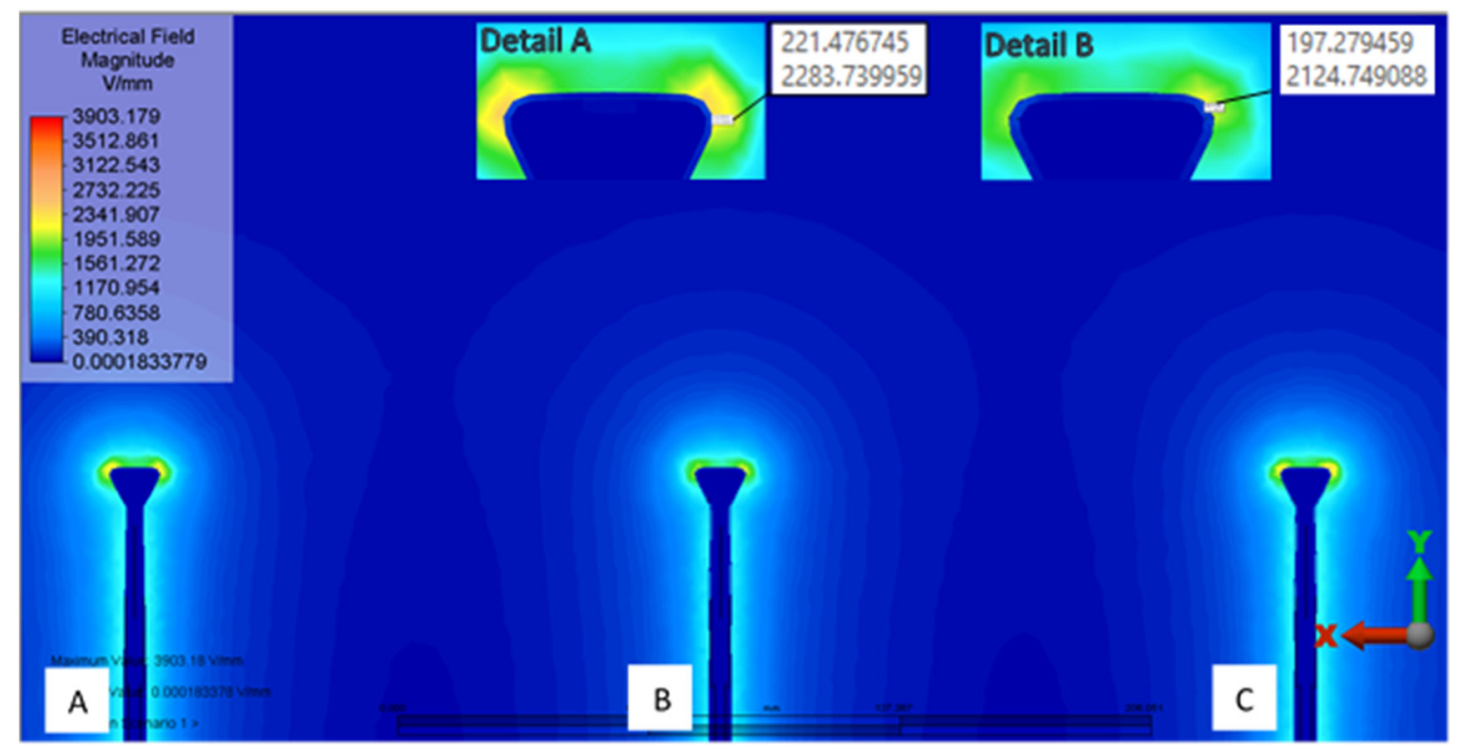

Figure 1 Distribution of electric field intensity around three electrodes, mutual distance $160 \mathrm{~mm}$

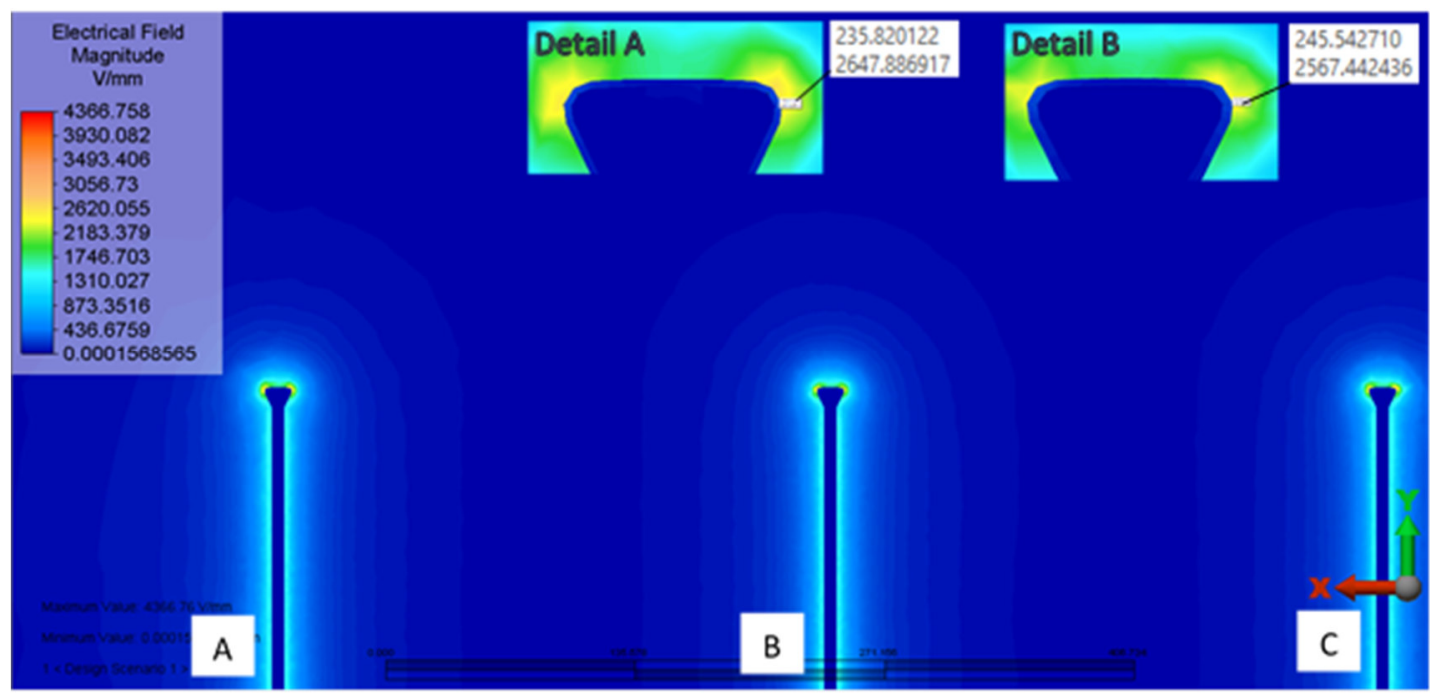

Figure 2 Distribution of electric field intensity around three electrodes, mutual distance $300 \mathrm{~mm}$

It can be seen from Figure 1 that at a distance of $160 \mathrm{~mm}$ among the electrodes, the maximum intensity on the electrode spinning surfaces decreases compared to the case where the distance is $300 \mathrm{~mm}$, see Figure 2. Details $A$ and $B$ (Figures 1 and 2) contain electric field values, furthermore, it is apparent from that there is a decrease in intensity on the inner sides of the outer electrodes (towards the middle electrode) compared to the outer sides. The intensity of the electric field on the surface of the middle electrode is also lower than on the outer electrodes. This occurs because of electrical influence of outer electrodes on the middle electrode. The detail $\mathrm{C}$ of the spinning electrode is not shown in the Figures 1 and $\mathbf{2}$, since the difference from the detail $A$ in both the values of the electric field and its distribution is negligible. Thus, the distribution of the 
electric field and its values around the outer electrodes $A$ and $C$ can be considered identical in all cases of simulations.

Figure 3 shows the graph showing the results of the simulations. The minimum technological value of the distance among the electrodes was $160 \mathrm{~mm}$ and with a $20 \mathrm{~mm}$ increment, a total of seven simulations were made up to an end distance of $300 \mathrm{~mm}$. The significant increase in intensity can be observed in the electrode spacing range of 160 to $200 \mathrm{~mm}$. The increase in intensity in the range from 200 to $300 \mathrm{~mm}$ is gradual and can be considered linear with neglected simulation inaccuracy. Another significant finding of the simulation is the same shape of the electric field intensity at the outer electrodes compared to the center electrode, but with the decrease of the intensity on the middle electrode.

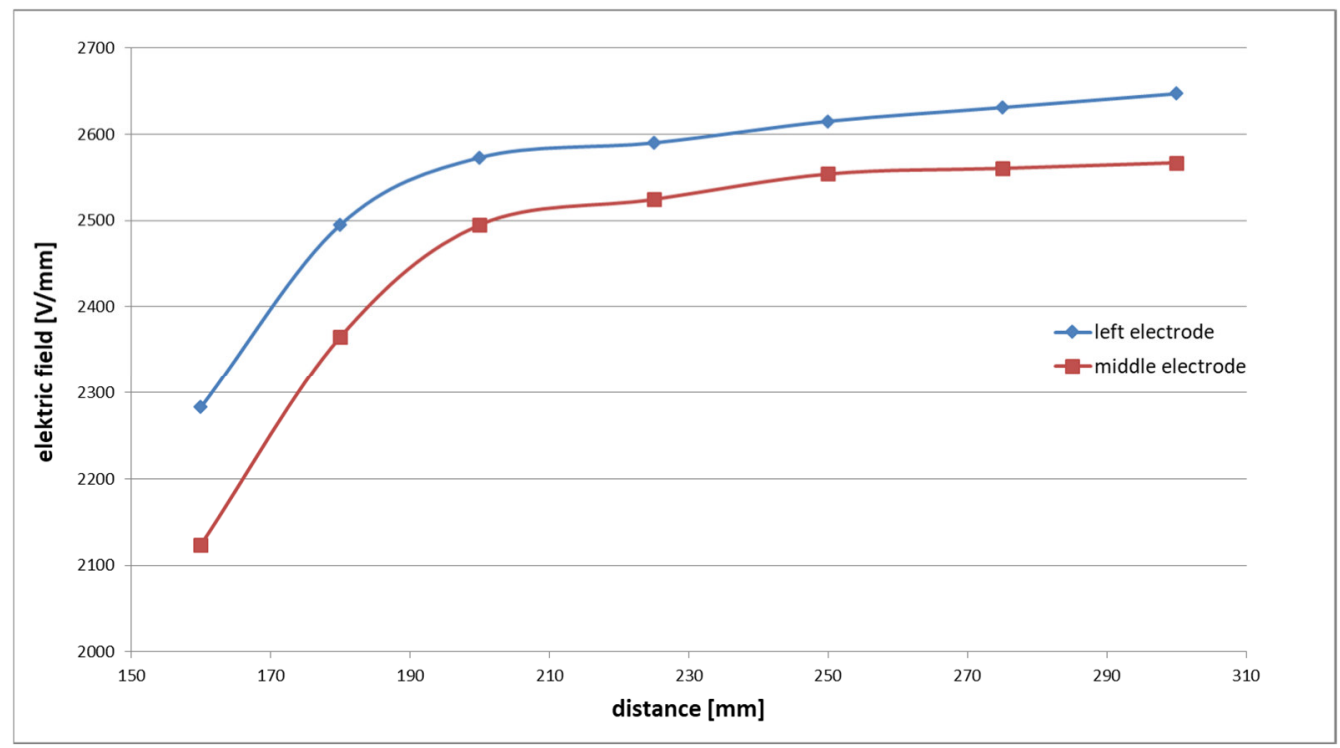

Figure 3 Dependence of electric field intensity distribution on electrode spacing

\section{EXPERIMENT}

The placement of the three electrodes 1, 2 and 3 (see Figure 4) was further tested directly in the spinning process. Electrode spacing variants with the same parameters as in the simulation were investigated (Chapter 2). The electrode power was supplied by AC electrical voltage with an effective value of $28.5 \mathrm{kV}$ and a frequency of $50 \mathrm{~Hz}$. A $10 \%$ solution of PVB Movital 60 polymer in ethanol was used for spinning. The temperature in the space, where the spinning process was performed reached $21^{\circ} \mathrm{C}$ and the humidity was $44 \%$. Figure 4 shows the testing process where a distance among electrodes was $160 \mathrm{~mm}$. The produced nanofiber stream was collected on the rotating cylinder on which the requested layer was formed. It can be stated that the nanofiber streams repulse at the electrode spacing of $160 \mathrm{~mm}$, especially at the outer electrodes, see Figure 4 . Figure 5 shows the minimum electrode spacing $(250 \mathrm{~mm})$ without significant repulsion and thus deflection of current nanofibers from the direct direction during electrospinning,

Furthermore, in the experiment, the production of the outer and the middle electrodes was analysed and compared at the above mentioned electrode distances (Chapter 2). The production was determined by measuring the weight of the samples of the resulting nanofibrous layer produced in a certain time. Samples were taken separately for each electrode and weighed independently. The production time was the same for all samples, 300 seconds. The results of the analysis revealed a trend in decreasing production with decreasing electrode spacing, this can be seen in the graph in Figure 6 . The graph shows that the middle electrode provides lower production than the outer electrodes, confirming the interaction observed in the experiments and also evaluated from the simulations performed. 


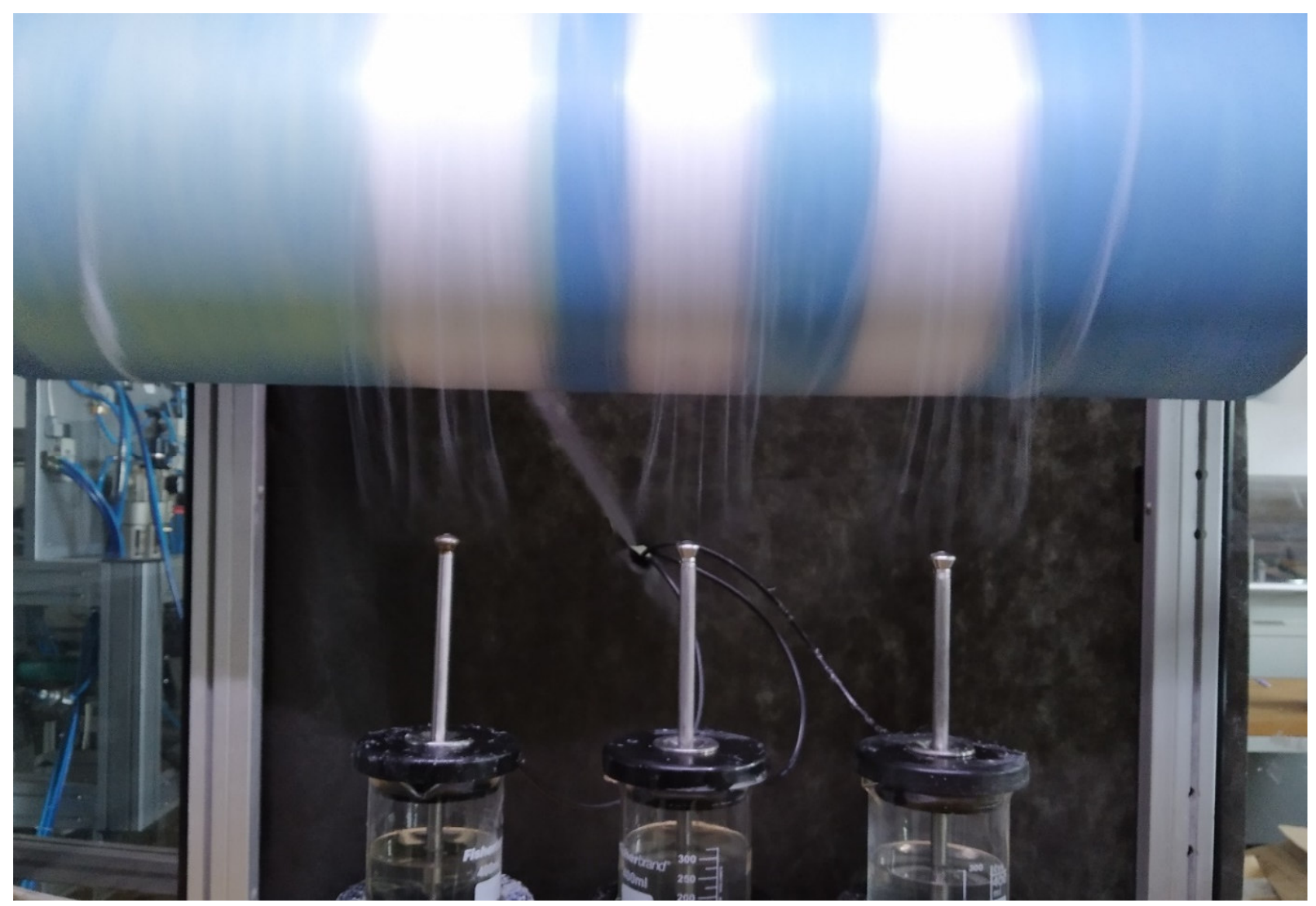

Figure 4 Electrospinning process, distance among electrodes is $160 \mathrm{~mm}$

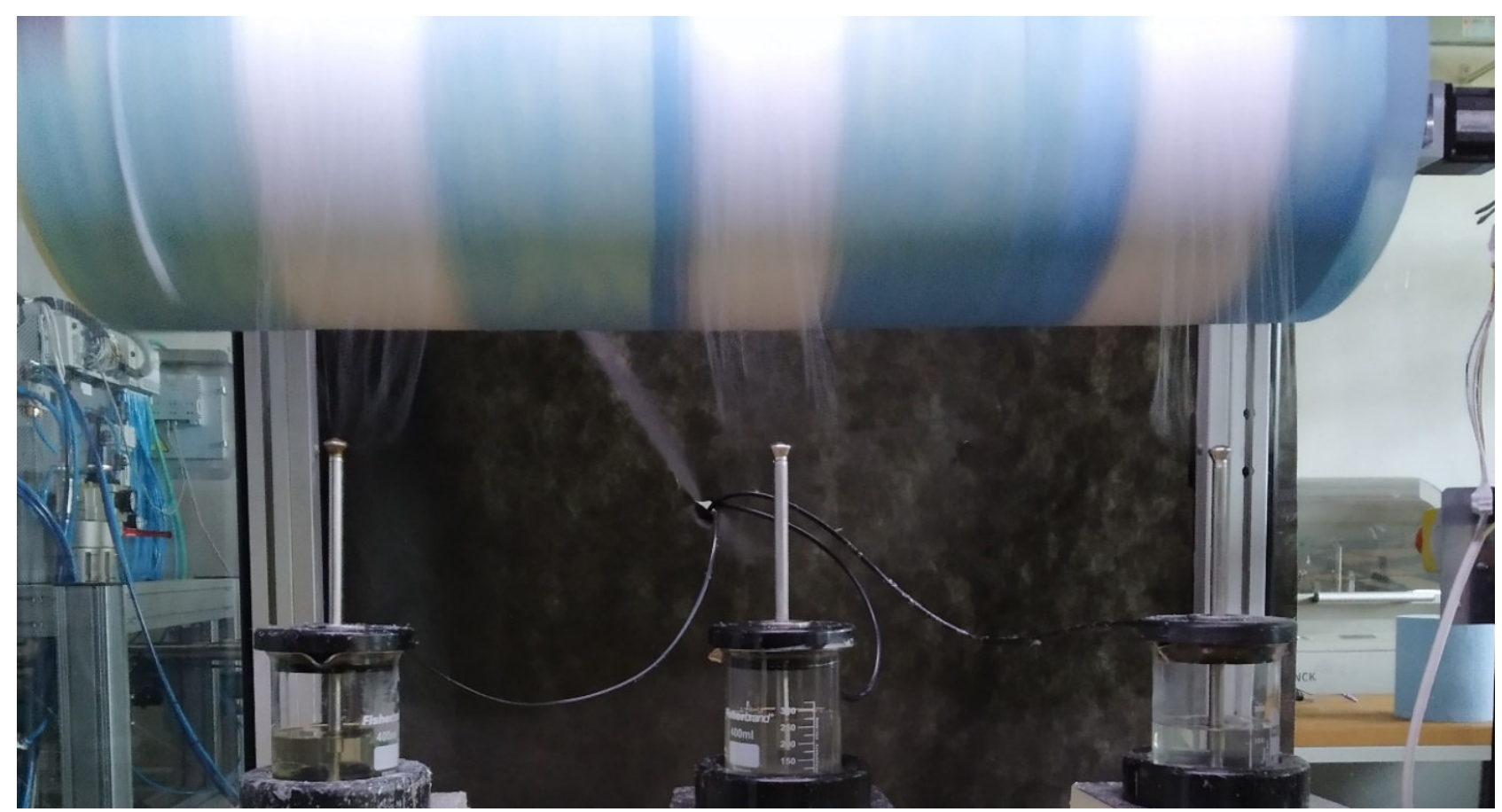

Figure 5 Electrospinning process, distance among electrodes is $250 \mathrm{~mm}$ 


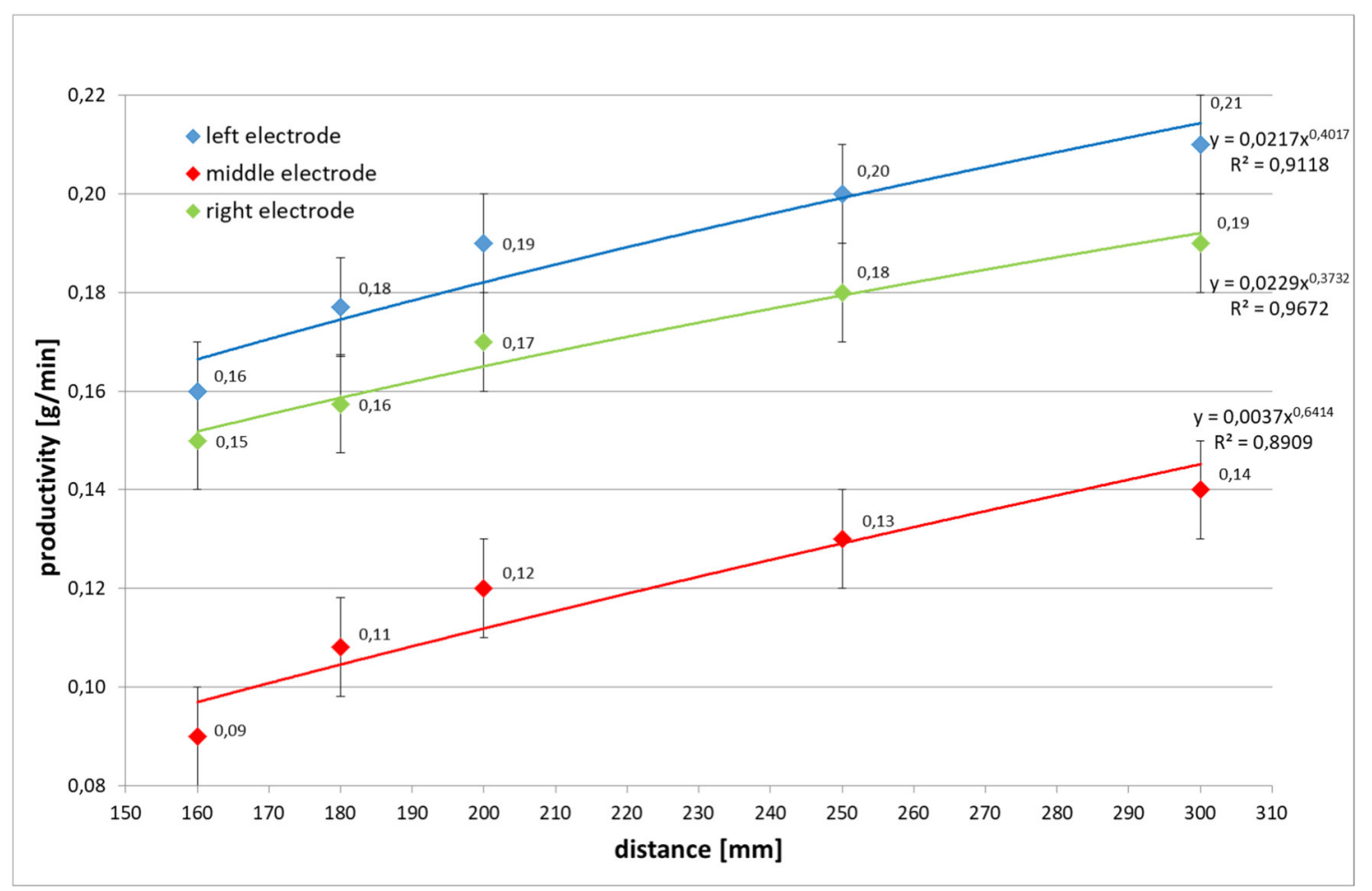

Figure 6 Electrode productivity dependence on distance among the electrodes

\section{CONCLUSION}

The aim of the work was to find out the interaction of the group of electrodes in one row with different distances. First, simulations were carried out to determine different distributions of electric field intensity around electrodes depending on their distance. The result was a change in the maximum intensity on the spinning surfaces of the electrodes. Figure 3 shows the decrease in intensity with decreasing electrode spacing, where the most significant decrease is observed in the area of electrode spacing from 160 to $200 \mathrm{~mm}$, the greatest intensity attenuation is on the middle electrode due to shading of the outer electrodes. The next step of the research was the realization of the experiment. The distance between individual electrodes was changed during testing. It has been observed that the above-described phenomena (repulsion of the nanofiber streams) are more intense as the electrodes converge towards each other. A comparison of the masses of nanofiber layers produced was observed a decrease in productivity on electrodes while decreasing their mutual distance. It has also been discovered that the middle electrode achieves 65 percent lower productivity than the outer electrodes.

\section{ACKNOWLEDGEMENTS}

This publication was written at the Technical University of Liberec as part of the project 21287 with the support of the Specific University Research Grant, as provided by the Ministry of Education, Youth and Sports of the Czech Republic in the year 2019.

\section{REFERENCES}

[1] RENEKER, D.H., YARIN, A.L., EVANS, E.A., KATAPHINAN, W., RANGKUPAN, R., LIU, W., KOOMBHONGSE, $\mathrm{S}$., $\mathrm{XU}, \mathrm{H}$. Electrospinning and nanofibers. In New frontiers in fiber science. 2001: Spring Conference. Raleigh, NC, USA, 2001, pp. 203-208. 
[2] SUN, Z. Compound Core-Shell Polymer Nanofibers Co-Electrospinning: Advanced Material. Binghamton: The Binghamton Press, 2003, pp.1929-1932.

[3] RŮŽIČKOVÁ, J. Electrostatic spinning of nano fibres, ISBN 80-7372-066-3, Liberec: Technical University of Liberec, 2006, p 11.

[4] POKORNY, P., (2014). Effective AC needleless and colector less electrospinning for yarn production. Physical Chemistry Chemical Physics, 2014, vol. 16, pp. 816-822.

[5] MACDIARMID, A.G., JONES, W.E., NORRIS, I.D., GAO, J., JOHNSON, A.T. JR., PINTO, N.J., HONE, J. , HAN, B., OKUZAKI, H., LLAGUNO, M. (2001). Electrostatically-generated nanofibers of electronic polymers: Synthetic Metals, Pennsylvania: University of Pennsylvania, 2001, p. 27.

[6] JIN, H.-J., FRIDRIKH, S.V., RUTLEDGE, G.C., KAPLAN, D.L. Electrospinning Bombyx mori Silk with (Polyethylene oxide). Biomacromolecules, American Chemical Society, 2002, p.408

[7] KOMAREK J., VALTERA J., SOUKUPOVA J., VYSLOUZILOVA L., SKRIVANEK J., ZABKA P., BERAN J., LUKAS. D. Verification of the mathematical model of the rod electrode in the electrospinning process. In Nanocon 2015, $7^{\text {th }}$ International Conference on Nanomaterials. Ostrava: TANGER, 2015. 